\title{
Numérisation de l'idéation : analyse des méthodes de travail collaboratif instrumenté en conception intégrée
}

\section{Digitalisation of ideation: Analysis of instrumented collaborative working methods in integrated design}

\author{
Gaëlle Baudoux*, Xaviéra Calixte et Pierre Leclercq \\ LUCID-ULiège, Allée de la Découverte 9, 4000 Liège, Belgique
}

\begin{abstract}
Résumé. Dans le contexte actuel de la conception architecturale, il s'avère utile de questionner l'instrumentation numérique de l'idéation. Dans cet article, nous étudions les méthodes de travail collaboratif instrumenté au travers des pratiques des concepteurs. En particulier, nous observons les "Moyens de conception", c'est-à-dire les types d'actions de conception mis en oeuvre et leurs articulations, indépendamment des logiciels utilisés. Nous pouvons ainsi mettre en évidence des combinaisons récurrentes de "Moyens", des patterns, témoins de leur complémentarité dans les activités, nous renseignant sur les pratiques et les besoins des concepteurs.
\end{abstract}

Mots-clés. Conception, Architecture, Numérique, Collaboration, Idéation, Moyen, Pratiques, Patterns.

\begin{abstract}
In the current context of architectural design, it seems usefull to question the articulation between the ideation at the beginning of the project and the BIM approach applied in the later phases. In this paper, we study collaborative working methods through the practices of designers. In particular, we observe the implemented "Means of design", i.e. the types of design actions, regardless of the software used. We can thus highlights recurring combinations of "Means", witnesses of complementarities between the activities of design. These complementarities inform us about designer's needs and usages.
\end{abstract}

Keywords. Design, Architecture, Digital, Collaboration, Ideation, Means, Usages, Patterns.

*Gaëlle Baudoux: gbaudoux@doct.uliege.be 


\section{Introduction}

Le BIM, Building Information Modeling, est la voie prometteuse prônée aujourd'hui pour multiplier les échanges entre acteurs et améliorer la performance des ouvrages bâtis. Cette démarche est certe bénéfique pour les phases de réalistation du projet mais se présente en inadéquation avec l'activité de conception en début de projet (NSCSC, 2010; LIST, 2015). En effet, celle-ci s'avère actuellement trop restrictive et trop rigide pour permettre au projet d'évoluer encore dans un sens créatif, ce qui est pourtant l'objectif principal de ces phases initiales.

Nous cherchons ici à mieux comprendre comment articuler ces premières phases d'idéation à la dynamique numérique contemporaine. Nous menons pour cela une étude préliminaire sur les activités d'idéation.

\section{Etat de l'art}

\subsection{Spécificités de la conception architecturale}

Le problème auquel doit répondre l'artefact architectural est complexe et mal défini (Borillo \& Goulette, 2002; Simon, 1969). Ainsi, il faut travailler en équipe. L'analyse et la résolution du problème se font en interaction, bien sûr la solution n'est jamais optimale mais plutôt un compromis satisfaisant. Toutefois l'évaluation de ces solutions ne peut se faire que par simulation mentale et, finalement, il n'y a pas de méthode de résolution prescrite (Darses, Détienne \& Visser, 2001; Safin, 2011). Dès lors, le concept, l'analogie, le dessin et la $3 \mathrm{D}$ prennent une place très importante dans le processus.

\subsection{Spécificités de la conception collaborative}

\subsubsection{Trois modes de conception collective}

La conception architecturale s'effectue bien souvent de manière collective (Darses, Détienne \& Visser, 2001; Rosenman \& Gero, 1999). Rapellons qu'il existe trois types de conception collective (Borillo \& Goulette, 2002; Darses \& Falzon, 1996; Elsen, 2011): la conception distribuée (coopération ou coopération faible), la co-conception (collaboration ou coopération forte) et la co-action. Une équipe de conception architecturale collaborative alterne entre des moments de conception distribuée et de co-conception (Ben Rajeb \& Leclercq, 2015).

\subsubsection{Activités cognitives spécifiques}

La conception collective mobilise des activités cognitives spécifiques, différentes de celles observées en conception individuelle. Les auteurs ci-avant les explicitent.

Ces nouvelles activités peuvent, tout d'abord, concerner la tâche. En effet, le problème est structuré collectivement au travers d'échanges pour identifier les buts et leurs dépendances et pour coordonner les tâches à venir; la génération et l'évaluation des solutions s'effectue collégialement, s'accompagnant d'argumentaires et de critiques et complexifiant ainsi la tâche suivant la différence de profil des concepteurs.

Ces nouvelles activités peuvent également toucher au processus. Les acteurs se synchronisent temporellement et opératoirement afin d'assurer la bonne gestion des tâches à réaliser; ils se synchronisent aussi cognitivement, de manière à coordonner les différents 
points de vue ou expériences, et ainsi créer respectivement un référentiel commun et un contexte de connaissances partagées, facilitant de ce fait les échanges.

Finalement, ces nouvelles activités peuvent être centrées sur l'interaction. Ainsi, pour que des acteurs puissent élaborer un projet, il est nécessaire, en plus de concevoir, qu'ils se coordonnent et qu'ils communiquent. De plus, les interactions créent de nouvelles dimensions de l'espace travail, la réflexion pouvant se faire individuellement, en aparté ou en groupe. Enfin, les acteurs se synchronisent temporellement sur l'organisation du travail, ce dernier pouvant être synchrone ou asynchrone.

\subsection{Moyens de conception}

En conception collaborative, nous faisons face à trois grand types d'action (Ellis \& Wainer, 1994): produire (i. e. caractériser l'objet architectural), communiquer (i. e. échanger des informations entre concepteurs à propos de l'objet) et se coordonner (i. e. organiser le travail collectif).

Dans cette étude, nous nous intéressons à l'action de produire et nous la caractérisons au travers des différentes actions effectuées dans ce but. Nous appelons ces différentes actions les moyens de conception ou "Moyens". Notons que nous ne nous attachons pas aux représentations produites via ces Moyens ni aux logiciels utilisés pour effectuer ces actions. Nous les synthétisons en 8 typologies d'actions, catégorisées en 8 mots-clefs (Anonyme, 2019). Nous retrouvons tout d'abord le Dessin-papier-main et le Dessin-numérique-main, qui désignent la conception via une production graphique à la main, respectivement sur support papier ou support numérique. Viennent également la DAO-2D et DAO-3D, faisant référence à la conception via une production graphique $2 \mathrm{D}$ ou $3 \mathrm{D}$ assistée par ordinateur. C'est à différencier de la CAO paramétrique qui symbolise la conception paramétrique assistée par ordinateur. Le mot-clef Maquette désigne la conception via production d'un modèle 3D physique et celui d'Image de référence, la conception par analogie à des représentations préexistantes. Finalement, nous regroupons toutes les conceptions par simulation numérique statique ou dynamique sous le terme Prototype.

\section{Problématique}

D'autres auteurs ont déjà travaillé sur le sujet des patterns ou de la conception collaborative comme Tang (2011) qui a utilisé le "function-behaviour-structure coding scheme" pour comparer différents environnements, Gero (1990) qui a analysé et codé le processus de conception au travers de "design prototypes" ou encore Ericsson et Simon (1993) qui se sont intéressés à l'évolution des objets et des outils indépendamment des situations d'usage. Mais ces études ne se penchent pas sur l'articulation entre les outils et les actions menées. Elles n'analysent pas non plus le processus dans sa globalité, contrairement à ce que nous nous proposons de faire.

Dans cet article, nous analysons l'usage des Moyens de conception en cherchant à dégager des patterns d'utilisation de ces Moyens, c'est à dire des combinaisons récurrentes de "Moyens", apparaissant dans les pratiques.

Cette analyse a fait l'objet d'une étude plus large visant à analyser des méthodes de travail collaboratif en conception architecturale intégrée, dans le but d'identifier les besoins des concepteurs de projets complexes en collaboration (Baudoux, 2019). Nous nous concentrons ici sur l'étude de ces patterns dans l'utilisation des Moyens. Quels patterns pouvons-nous observer? Quels rôles ont-ils dans les pratiques des concepteurs ? A quels besoins répondent-ils? 


\section{Méthodologie}

\subsection{Méthode de recherche}

Pour répondre à cette problématique, et récolter des données sur les activités des concepteurs, nous avons instrumenté un processus de conception. Celui-ci prend place dans le contexte d'un atelier de master, ayant pour objectif la conception collaborative, durant 14 semaines et avec 8 équipes de 3 à 4 concepteurs, d'un avant-projet de musée de $7500 \mathrm{~m} 2$.

La récolte des données est basée sur le remplissage régulier de fiches-résumés du projet par les concepteurs et leur questionnement sur les pratiques mises en place au travers de questionnaires. Une fois les données recueillies, codées et les formalismes visuels générés, nous avons procédé à plusieurs analyses descriptives graphiques pour dégager des tendances dans les pratiques. Nous avons ainsi pu identifier un certain nombre de patterns apparaissant plus ou moins souvent suivant le moment de processus. Nous avons également dégagé différentes raisons de choix des Moyens. Nous avons ensuite questionné les tendances observées en regard de la littérature et du ressenti des usagers.

\subsection{Méthode de récolte des données}

Nous expliquons ici la méthode par questionnaire ayant permis de récolter les données nécessaires à la génération des résultats présentés dans cet article. La méthode en ellemême ayant déjà fait l'objet d'un article spécifique (Baudoux et al., 2019), nous l'expliciterons donc brièvement.

Le questionnaire est rempli par chacun des 27 concepteurs en 5 moments-clefs de son processus. Ces moments correspondent à des étapes de sous-objectifs accomplis, comme notamment la répartition fonctionnelle, le design de la structure, ... Dans le cadre de l'atelier, les revues de projet avec des experts marquent ces sous-étapes. Le questionnaire interroge le concepteur sur les Moyens utilisés en lui demandant de les citer par ordre d'importance, d'évaluer la pertinence de leur usage, d'expliquer en quelques mots les raisons de cette évaluation et les raisons de choix de ces Moyens. Si l'un ou l'autre Moyen s'avère moins pertinent, les concepteurs sont invités à proposer une alternative plus appropriée à leur objectif de conception et à expliquer en quelques mots les raisons de ce choix.

\section{Résultats}

\subsection{Identification des patterns}

Nous partons des données détaillant les Moyens choisis par ordre d'importance par chaque concepteur, récoltées au moyen du questionnaire. Après codage, nous obtenons les graphiques suivants (Figure 1) pour chacune des séquences observées, chaque colonne représentant un ordre d'importance du premier au cinquième.

Ces formalismes visuels permettent de recenser l'ensemble des combinaisons de Moyens qui apparaissent tout au long du processus. Nous identifions 12 combinaisons différentes. Parmi toutes ces combinaisons, nous ne retenons que celles apparaissant plus de $8 \%$ des fois sur l'ensemble du processus ou $15 \%$, et plus, des fois sur une séquence particulière. Cela nous permet d'écarter les combinaisons très peu présentes et donc représentatives de pratiques vraiment marginales. Notons que certaines combinaisons présentent un groupement de deux Moyens, additionnés, quelques fois, d'un troisième. Ce troisième est retenu dans le pattern s'il apparaît chez au moins un tiers des concepteurs utilisant la combinaison initiale. 


\begin{tabular}{|c|c|c|c|c|c|}
\hline Acteur Z & Dessin papier & DAO 3D & & & \\
\hline Acteury & Dessin papier & DAO 3D & & & \\
\hline Acteur X & Dessin papier & DAO 3D & Autre & & \\
\hline ActeurW & Dessin papier & $\mathrm{DAO} 3 \mathrm{D}$ & Autre & & \\
\hline ActeurV & Dessin papier & & Image référence & & \\
\hline Acteuru & DAO 3D & Dessin papier & Autre & & \\
\hline Acteur T & DAO 3D & Dessin papier & Autre & Image référence & \\
\hline Acteurs & DAO 3D & Dessin papier & $\mathrm{DAO} 2 \mathrm{D}$ & CAO param & Maquette \\
\hline$\overline{\text { Acteur R }}$ & Dessin papier & DAO 3D & DAO $2 \bar{D}$ & & \\
\hline$\overline{\text { Acteur Q }}$ & Image référence & Dessin papier & DAO 3D & & \\
\hline ActeurP & $\mathrm{DAO}$ 3D $\mathrm{De}$ & ssin papier & & & \\
\hline Acteuro & Dessin papier & & & & \\
\hline Acteur $\mathrm{N}$ & Dessin papier & & & & \\
\hline Acteur M & Dessin papier & Dessin num & Image référence & & \\
\hline ActeurL & Dessin papier & Image référence & & & \\
\hline Acteurk & Dessin papier & Image référence & & & \\
\hline Acteur J & Dessin papier & Image référence & & & \\
\hline Acteurl & Dessin papier & Dessin num & Image référence & & \\
\hline Acteur H & Dessin papier & DAO 3D & & & \\
\hline Acteur G & Dessin papier & $\mathrm{DAO} 2 \mathrm{D}$ & DAO 3D & & \\
\hline ActeurF & Dessin papier & $\overline{D A O} 3 \mathrm{D}$ & & & \\
\hline Acteur E & Dessin papier & Image référence & DAO 3D & & \\
\hline Acteur D & Dessin papier & & & & \\
\hline Acteur C & Dessin papier & Image référence & & & \\
\hline Acteur B & Dessin papier & DAO 3D & férence & & \\
\hline Acteur AA & Dessin papier & DAO 3D & & & \\
\hline Acteur A & $\overline{D A O}$ 3D & DAO 2D & Maquette & & \\
\hline
\end{tabular}

Figure 1. Ensemble des Moyens choisis par chaque concepteur, classés du premier au cinquième par ordre d'importance, ici pour la séquence 1 , et identification du pattern $n^{\circ} 1$.

Des combinaisons observées, nous pouvons donc identifier 4 types de patterns (Figure 2). Le premier pattern (DESS_PAP + DAO_3D) allie le dessin à la main sur un support papier au dessin assisté par ordinateur en trois dimensions. Il s'adjoint parfois du dessin assisté par ordinateur en deux dimensions. Le second pattern (DESSIN_PAP + IMAGE) est composé du dessin à la main sur un support papier suivi, dans l'importance, par l'utilisation d'images de référence. Le troisième pattern (DAO_3D + DAO_2D) associe le dessin assisté par ordinateur en trois dimensions avec le dessin assisté par ordinateur en deux dimensions, auquels s'ajoute chez certains concepteurs la réalisation d'une maquette. Finalement, le quatrième pattern (DESS_PAP + DAO_2D) combine le dessin à la main sur un support papier et le dessin assisté par ordinateur en deux dimensions. Ces deux moyens sont parfois complétés par la DAO 3D.

Pattern 1

Dessin papier

DAO 3D

Pattern 1bis

DAO 3D Dessin papier

\section{Pattern 2}

Dessin papier $\quad$ Image Référence

\section{Pattern 3}

$$
\text { DAO 3D }
$$

Pattern 4

DAO 2D

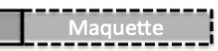

Pattern 3bis

DAO 2D DAO 3D

Dessin papier DAO 2D 1 DAO 3D --

Figure 2. Synthèse des différents types de patterns identifiés. 
Notons que nous étudions séparément les patterns et leur inverse (dénommé "bis"). En effet, à ce stade de la recherche, nous ne savons pas si l'ordre des Moyens dans la combinaison aura un impact sur les résultats.

\subsection{Fréquence d'utilisation}

La comptabilisation du nombre de patterns employés par chaque concepteur nous mène au graphique suivant (Figure 3). Nous y mettons en évidence l'occurrence de chaque pattern avec, en parallèle, la thématique de la revue dispensée au terme de la séquence observée. Cette thématique conditionne en effet en partie les objectifs de chaque séquence. Elle peut donc influencer le type de pattern mobilisé.

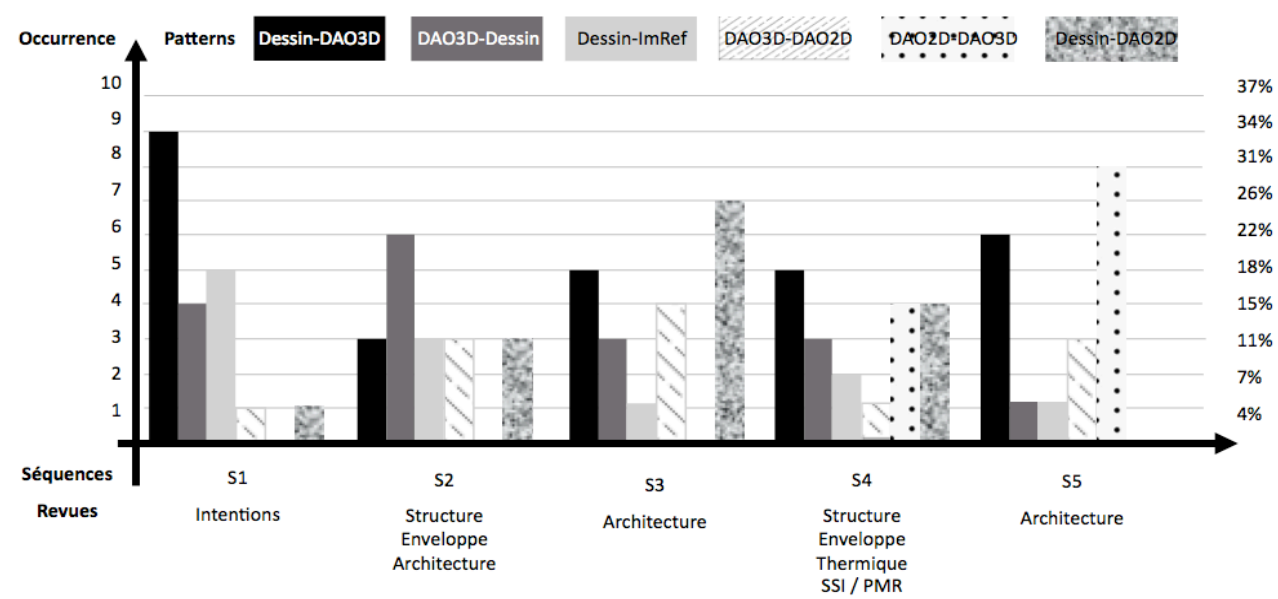

Figure 3. Pourcentage d'occurrence des différents patterns.

Nous pouvons ainsi voir que le pattern 1 (DESS_PAP + DAO_3D), en noir dans le graphique, est largement employé en séquence 1. Il se démarque nettement des autres patterns. Dans la séquence 2, par contre, tous les patterns convergent vers une même fréquence d'utilisation. A l'exception du pattern 1bis (DAO_3D + DESS_PAP, en gris foncé) qui présente un emploi assez important, et du pattern 3bis (DAO_2D + DAO_3D, en pois) qui ne sera pas du tout utilisé ainsi que dans les trois premières séquences.

Le pattern 1 (DESS_PAP + DAO_3D) présente, après cette séquence, une fréquence d'usage de plus en plus importante. De même, le pattern 3bis (DAO_2D + DAO_3D) prend son essor à la troisième séquence pour finir, en séquence 5, par être le pattern principalement utilisé.

Le pattern 2 lui (DESS_PAP + IMAGE, en gris clair) montre globalement une tendance décroissante tout au long des séquences, tout comme le pattern 1bis (DAO_3D + DESS_PAP). Tandis que le pattern 4 (DESS_PAP + DAO_2D, en marbré) croît jusqu'à la séquence 3 puis décroît au fur et à mesure des séquences restantes.

Finalement, le pattern 3 (DAO_3D + DAO_2D), en hachuré, présente une variation irrégulière et de faible amplitude. 


\section{Discussion}

\subsection{Fréquence d'utilisation}

Une fois les fréquences d'utilisation relevées (point 5.2), nous cherchons à comprendre les raisons pour lesquelles certains patterns sont mis en place dans des séquences préférentielles. Pour cela, non seulement, nous étudions les fréquences d'utilisation des différents types de patterns identifiés et les différentes raisons de choix que nous avons établi au vu des questionnaires pour les patterns concernés, mais nous analysons aussi les rôles des différents Moyens exprimés dans la littérature (Safin, 2011; Elsen, 2011). Nous les mettons en perspective avec les objectifs propres de chaque revue balisant les séquences observées. Nous réalisons cette analyse séquence par séquence.

Ainsi nous observons que le pattern 1 (DESS_PAP + DAO_3D) est très souvent utilisé lors de l'idéation et diminue avec celle-ci. Le Dessin-papier reste, toutefois, utilisé tout au long du processus tandis que la DAO-3D devient systématiquement associée, au fur et à mesure de la concrétisation des idées au fil des séquences. L'utilisation de ce pattern en ordre inverse (DAO_3D + DESS_PAP), quant à lui, diminue au fur et à mesure de la conception et donc de la concrétisation de l'objet.

Le pattern 2 (DESS_PAP + IMAGE), dans lequel l'Image-de-référence complète le Dessin-papier, est préférentiellement employé lorsque de nouvelles idées encore au stade d'ébauche sont générées c'est-à-dire lorsque les revues abordent une nouvelle thématique. Le pattern 3 (DAO_3D + DAO_2D) n'est pas utilisé préférentiellement vis-à-vis d'objectifs particuliers. La Maquette y est néanmoins adjointe uniquement lorsque les objectifs des revues touchent une nouvelle thématique, amenant ainsi un besoin d'idéation itérative. Pour le pattern inverse (DAO_2D + DAO_3D), il est employé lorsque l'artefact est caractérisé de façon formelle et précise, dans le but de fournir les rendus normalisés attendus, ainsi que lorsqu'il est évalué en 3D pour juger des solutions finales.

Finalement pour ce qui est du pattern 4 (DESS_PAP + DAO_2D), il est le plus mis en œuvre en milieu de conception puisqu'à ce moment le concepteur caractérise l'objet sous forme de plans et coupes, et l'évalue également, tout en ayant toujours besoin de représenter facilement les idées fraîchement générées.

\subsection{Rôle des patterns}

Pour interroger les rôles des patterns, nous partons des données récoltées grâce au questionnaire sur les raisons de choix des différents Moyens. Une fois les données traitées, nous pouvons lister, par ordre d'occurrence, l'ensemble des raisons invoquées par les concepteurs lors du choix de chaque Moyen.

Pour chaque pattern identifié, nous comparons les listes de raisons de choix relatives aux différents Moyens mobilisés dans ce pattern. Nous cherchons alors à identifier des complémentarités dans les raisons de choix qui pourraient expliquer l'utilisation groupée de ces Moyens. Cette interprétation se fait également en regard des rôles des Moyens exprimés dans la littérature (Elsen, 2011; Visser, 2001).

Nous complétons cette analyse (Figures 4 à 9) par de courts entretiens avec les concepteurs afin de valider les résultats formulés. Cet entretien synthétise chaque hypothèse de raison de choix d'un pattern et demande au concepteur si cette complémentarité est bien la raison de choix du pattern. Les dires des concepteurs convergent dans le même sens que nos interprétations à l'exception de l'usage de la maquette dans le pattern 3 . Nous pouvons donc conclure quant aux complémentarités entre les Moyens associés en patterns et donc quant aux raisons de choix de ces patterns. 


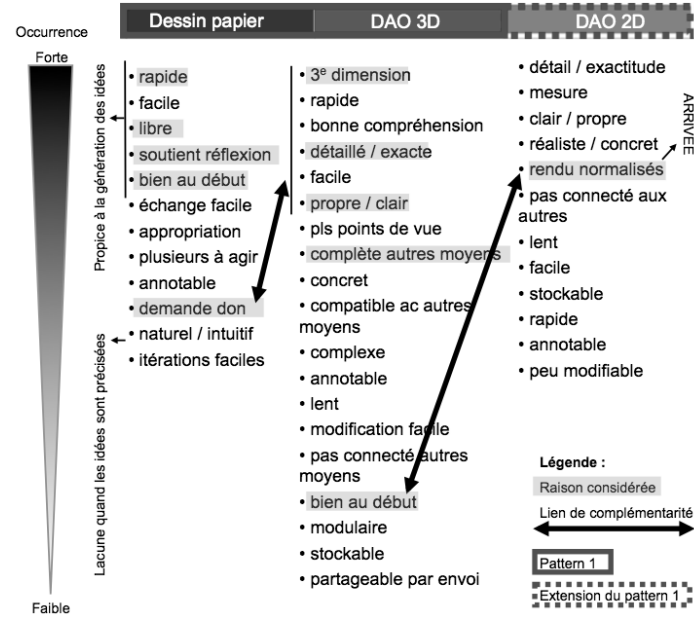

Figure 4. Complémentarité entre les raisons de choix du pattern $n^{\circ} 1$.

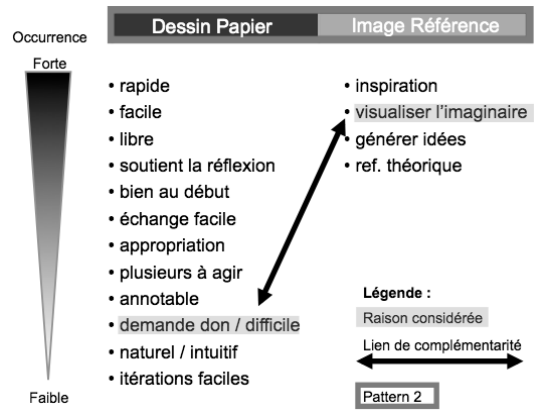

Figure 6. Complémentarité entre les raisons de choix du pattern $n^{\circ} 2$.

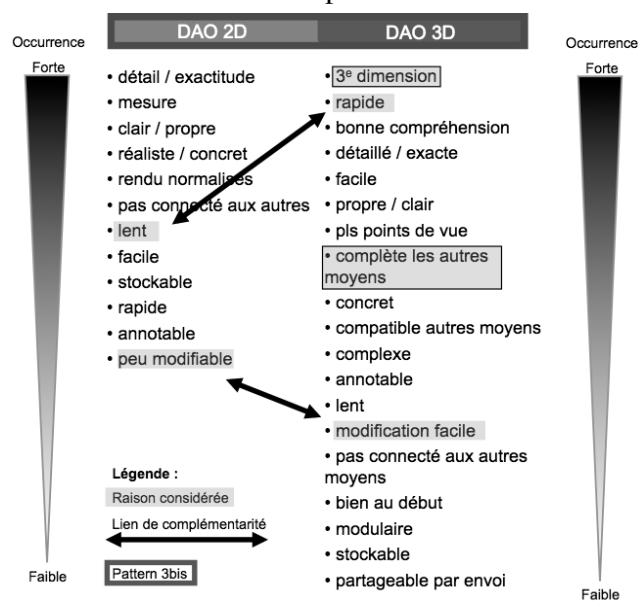

Figure 8. Complémentarité entre les raisons de choix du pattern $n^{\circ} 3$ bis.

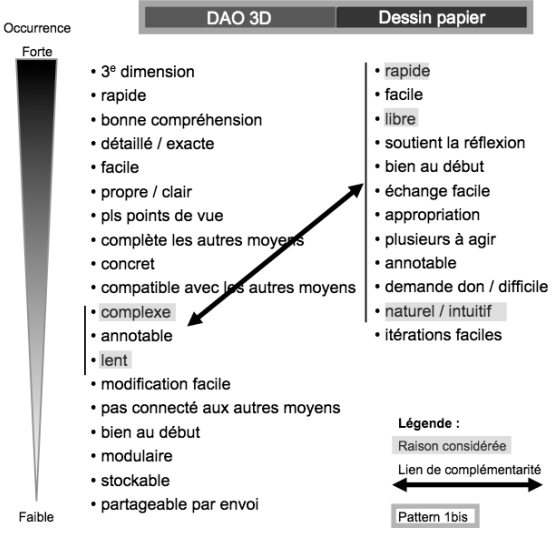

Figure 5. Complémentarité entre les raisons de choix du pattern $n^{\circ} 1$ bis.

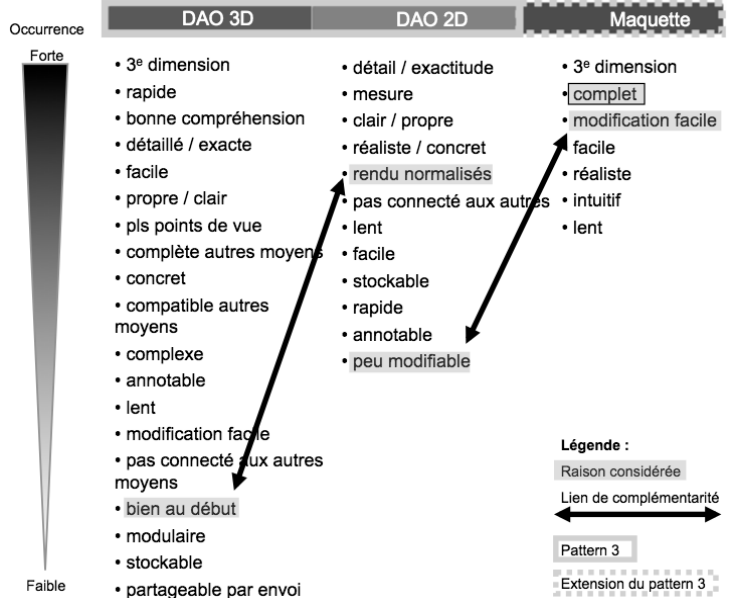

Figure 7. Complémentarité entre les raisons de choix du pattern $n^{\circ} 3$.

\begin{tabular}{|c|c|c|}
\hline Dessin Papier & DAO 2D & \\
\hline $\begin{array}{l}\text { - rapide } \\
\text { - facile } \\
\text { - libre } \\
\text { - soutient la réflexion } \\
\text { - bien au début } \\
\text { - échange facile } \\
\text { - appropriation } \\
\text { - plusieurs à agir } \\
\text { - annotable } \\
\text { - demande don / difficile } \\
\text { - naturel / intuitif } \\
\text { - itérations faciles }\end{array}$ & $\begin{array}{l}\text { - détail / exactitude } \\
\text { - mesure } \\
\text { - clair / propre } \\
\text { - réaliste / concre } \\
\text { - rendu norm fisés } \\
\text { - pas cop/ecté autre } \\
\text { - lent } \\
\text { - facile } \\
\text { - stockable } \\
\text { - rapide } \\
\text { - annotable } \\
\text { - peu modifiable }\end{array}$ & $\begin{array}{l}3^{e} \text { dimension } \\
\text { - rapide } \\
\cdot \text { bonne compréhension } \\
\text { - détaillé / exacte } \\
\text { - facile } \\
\text { - propre / clair } \\
\text { - pls points de vue } \\
\begin{array}{l}\text { - complète les autres } \\
\text { moyens }\end{array} \\
\text { - concret } \\
\text { - compatible ac autres } \\
\text { moyens } \\
\text { - complexe } \\
\text { - annotable } \\
\text { - lent } \\
\text { - modification facile } \\
\text { - pas connecté autres } \\
\text { moyens } \\
\text { - bien au début } \\
\text { - modulaire } \\
\text { - stockable } \\
\text { - partageable par envoi }\end{array}$ \\
\hline
\end{tabular}

Figure 9. Complémentarité entre les raisons de choix du pattern $n^{\circ} 4$. 
Ainsi, en conclusion, pour le pattern 1 (DESS_PAP + DAO_3D), la DAO-3D est utilisée en complément du Dessin-papier parce qu'elle supplée le dessin par sa clarté et ses détails lorsque les idées se compliquent. Elle est parfois complétée à son tour par la DAO2D, seul Moyen permettant de produire les rendus normalisés demandés en fin de projet. A l'inverse (DAO_3D + DESS_PAP), le Dessin-papier est associé à la DAO-3D parce qu'il est plus rapide, libre et intuitif. Dans le pattern 2 (DESS_PAP + IMAGE), les Images-deréférence permettent de visualiser les idées difficilement dessinables. Pour le pattern 3 (DAO_3D + DAO_2D), comme dit plus haut, la DAO-2D est utilisée en complément de la DAO-3D afin de produire les rendus normalisés nécessaires. En sens inverse (DAO_2D + DAO_3D), la DAO-3D peut également compléter la DAO-2D en apportant la possibilité de représenter la 3e dimension. Elle est, en outre, plus rapide et plus facilement modifiable lorsque les idées évoluent promptement. Finalement concernant le pattern 4 (DESS_PAP + DAO_2D), la DAO-2D complète le Dessin-papier en étant plus claire, exacte et concrète.

\section{Conclusion}

Dans ce travail, nous avons cherché à identifier des patterns dans l'utilisation des Moyens de conception et nous avons formulé des hypothèses d'explications quant à leur rôle dans les pratiques des concepteurs et aux besoins de conception auxquels ils répondent.

Pour cela, nous avons suivi durant 4 mois un processus de conception collaborative intégrée en milieu pédagogique. Une fois les données récoltées grâce à notre méthode par questionnaire, nous avons pu identifier 4 patterns différents et suffisamment utilisés pour être représentatifs de pratiques de conception. Nous avons également calculé les fréquences d'utilisation de chacun de ces patterns. Nous avons ensuite pu formuler des hypothèses expliquant les complémentarités entre les Moyens associés en patterns en examinant les complémentarités décelées entre les raisons de choix énoncés par les concepteurs. Ces complémentarités ont été confirmées par ceux-ci lors d'entretiens post-conception. En nous référant aux objectifs des différentes séquences, aux rôles des patterns et aux rôles des Moyens évoqués dans la littérature, nous avons pu identifier des justifications potentielles à propos des fréquences observées.

Questionnant l'articulation entre l'idéation du début de projet et les approches numériques qui seront mises en oeuvre par le BIM dans les phases ultérieures, nous avons ainsi pu qualifier les méthodes de travail collaboratif au travers des pratiques des concepteurs, permettant de mieux comprendre la phase d'idéation en projet, coeur de la recherche qui nous anime.

Diverses limites ont été rencontrées lors de cette étude. La mise en place du questionnaire a dû être accompagnée d'explications, à la fois pour les concepts observés mais aussi sur les termes présents dans les questions. Lors de l'étude du rôle des patterns, les raisons de choix ont été étudiées uniquement qualitativement. Nous nous concentrions en effet sur le vécu des concepteurs et sur leurs pratiques, amenant donc naturellement à une analyse qualitative.

En termes de perspectives, nous projetons d'étudier plus en profondeur l'usage des patterns identifiés, en analysant leur impact sur l'efficacité des Moyens employés ainsi que sur le processus, par l'observation de deux variables : l'avancement du projet et la stabilité des idées. Les données recueillies permettent également d'étudier les pratiques à travers d'autres questionnements portant, par exemple, sur le détournement des Moyens et leur appropriation par utilisateur ou portant sur le respect du processus spontané du concepteur.

Dans le contexte architectural actuel, il s'avère utile de questionner l'articulation entre l'idéation du début de projet et la démarche BIM appliquée à sa suite. Les observations 
présentées ici constituent une première étude pour mieux comprendre l'idéation et pouvoir envisager la question de la transition. Nous en retirons nombre d'informations, notamment sur les activités mise en place dans le processus de création, les types de Moyens employés et à quel moment et les besoins des concepteurs en terme d'instrumentation.

\section{Bibliographie}

Baudoux, G. (2019). Analyse des méthodes de travail collaboratif en conception intégrée: analyse des patterns d'usage des Moyens de conception. Master Thesis, University of Liège, Belgium.

Baudoux, G., Calixte, X. \& Leclercq, P. (2019). Analysis of instrumental practices in collaborative design: method of identifying needs, means and their effectiveness. 16th International Conference on Cooperative Design, Vizualization and Engineering, CDVE 2019, Spain, Mallorca, 2019.

Ben Rajeb, S., \& Leclercq, P. (2015). Co-construction of meaning via a collaborative action research approach. Yuhua Luo éd., In LNCS, Lecture Notes in Computer Sciences, Cooperative Design, Visualization, and Engineering, 9320, pp. 205-215.

Borillo, M., Goulette, J.P. (2002). Cognition et création : Explorations cognitives des processus de conception. Sprimont : Mardaga

Darses, F., Détienne, F., \& Visser, W. (2001). Assister la conception: perspectives pour la psychologie cognitive ergonomique. Paper presented at the E'PIQUE 2001, Actes des journées d'étude en psychologie ergonomique.

Darses, F., Falzon, P. (1996). La conception collective : une approche de l'ergonomie cognitive. In G. de Terssac \& E. Friedberg (Eds). Coopération et Conception. Toulouse : Octarès.

Ellis, C., Wainer, J. (1994). A conceptual model of groupware. Chapel Hill, NC

Elsen, C. (2011). La médiation par les objets en design industriel, perspectives pour l'ingénierie de conception. PhD Thesis, University of Liège, Belgium

Ericsson, K.A. \& Simon, H.A. (1993). Protocol analysis: Verbal reports as data, MIT Press, Cambridge.

Gero, J.S. (1990). Design prototypes: A knowledge representation, schema for design. In AI Magazine, 11(4), pp. 26-36.

LIST, Luxembourg Institute of Science \& Technologie (2015). Récupéré sur la méthode BIMetric : http://bimetric.list.lu/

NSCSC, Nova Scotia Construction Sector Council, Industrial Commercial Institutional (2010). Functional Information Technology Phase 1: Detailed Analysis, préparé par le Construction Engineering and Management Group de l'Université du Nouveau Brunswick.

Rosenman, M.A., \& Gero, J.S. (1999). Purpose and function in collaborative CAD environment. In Reliability Engineering and System Safety, 64(2), pp. 167-179.

Safin, S. (2011). Processus d'externalisation graphique dans les activités cognitives complexes :le cas de l'esquisse numérique en conception architecturale individuelle et collective. PhD Thesis, University of Liège, Belgium.

Simon, H.A. (1969). The Science of the Artificial. Cambridge, MA : MIT Press

Tang, H.H., Lee, Y.Y. \& Gero, J.S. (2011). Comparing collaborative co-located and distributed design processes in digital and traditional sketching environments: A protocol study using the function-behaviour-structure coding scheme. In Design Studies, 32(1), pp. 1-29.

Visser, W. (2001). Conception individuelle et collective. Approche de l'ergonomie cognitive. Le Chesnay, France: INRIA - Institut national de la Recherche en Informatique et Automatique. 